\title{
SUCCESeR: Simple and Useful Multi Color Concepts for Effective Search and Retrieval
}

\author{
Satishkumar L. Varma \\ Department of Computer Engineering \\ Pillai's Institute of Information Technology \\ Navi Mumbai, India
}

\author{
Sanjay N. Talbar \\ Electronics and Telecommunication Engineering \\ SGGS Institute of E\&IT \\ Nanded, India
}

\begin{abstract}
The image quality depends on level of intensities used in images. Image is consists of various types of objects. Objects in the images are distinguishable because of various intensity levels used. The concentration of intensity levels so called energy can be extracted from image using discrete cosine transform (DCT). In this paper we apply DCT 8x8 block coefficients separately on three different color planes of three different color models namely RGB, HSV and YCbCr. The different elements of ten DCT coefficient matrices are used to form feature vectors. The different feature vectors are formed using these ten elements. These feature vectors are used to index all images in the database. The system was tested with Coral Image database containing 1000 natural images having 10 different classes of images. The image retrieval using these indices is giving comparatively better results.
\end{abstract}

Keywords-color model; discrete cosine transform; image indexing; image retrieval;

\section{INTRODUCTION}

With the explosive growth of the need of the information, the large amount of storage, manipulation and transmission of data also needs to be kept indexed.

Content Based Image Retrieval (CBIR) is a technique which uses visual contents in an image to retrieve images. The visual contents are called features. These features are used to search images from large scale image databases according to users' requests in the form of a query image [1], [8], [9].

The system is tested with Coral Image database used in [8] containing 1000 natural images having 10 classes of images. These images have also been used in SIMPLIcity [7], Columbia VisualSEEK and WebSEEK [16], and Stanford WBIIS [16].

\section{A. Related Work and Systems}

In the commercial domain, IBM QBIC [19] is one of the earliest systems. Recently, additional systems have been developed at IBM T.J. Watson [20], VIRAGE [21], NEC AMORA [22], Bell Laboratory [23], and Interpix. In the academic domain, MIT Photobook [24], [25 [26] is one of the earliest. Berkeley Blobworld [27], Columbia VisualSEEK and WebSEEK [16], CMU Informedia [28], UCSB NeTra [29], UCSD [30], University of Maryland [31], Stanford EMD [32], and Stanford WBIIS [16] are some of the recent systems. For example, [2] describes a method for image retrieval purely based on color and texture.

Ngo et al. developed an image indexing algorithm via reorganization of DCT coef-ficients in Mandala domain, and representation of color, shape and texture features in compressed domain [13]. Feng et al. proposed an indexing approach by direct extrac-tion of statistic parameters in DCT domain to combine the nature of texture and shape into an integrated feature [14]. Ladert and Guerin-Dugue proposed an algorithm for extracting the global distribution of local dominant orientation from DCT domain [15].

\section{B. Contribution and Paper Organization}

The major contribution of this paper is not only to suggest feature extraction method to index images for faster and efficient digital image retrieval but also propose a clearly designed architecture that can be used for image classification.

The paper is organized as follows. In the following Section II the three color spaces and there conversion from one space to another is presented. The very next Section III explains the proposed CBIR model for image indexing and retrieval. The experimental results are discussed in Section IV. The summary and conclusion of the paper is presented in Section V. The future scope is suggested in Section VI.

\section{COLOR MODEL}

Color is perhaps the most expressive of all the visual features and has been extensively studied in the image retrieval research during the last decade.

\section{A. RGB Color Model}

The quantity of light is generated by the pixel results from the sum of Red, Green and Blue that are stated by the computer. The color model is shown in Fig. 1.

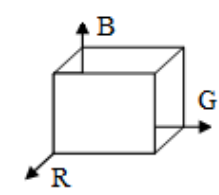

Figure 1. RGB Color Space 


\section{B. HSV Color Model}

Let $\mathrm{r}, \mathrm{g}, \mathrm{b}[0,1]$ be the red, green, and blue coordinates, respectively, of a color in RGB space. Let max be the greatest of $\mathrm{r}, \mathrm{g}$, and $\mathrm{b}$, and min the least. To find the hue angle $\mathrm{h}[0$, 360] for either HSI or HSV space, compute:

$$
h=\left\{\begin{array}{cl}
0 & \text { if } \max =\min \\
\left(60^{\circ} \times \frac{g-b}{\max -\min }+0^{0}\right) \bmod 360^{\circ}, & \text { if } \max =r \\
60^{\circ} \times \frac{b-r}{\max -\min }+120^{\circ}, & \text { if } \max =g \\
60^{\circ} \times \frac{r-g}{\max -\min }+240^{\circ}, & \text { if } \max =b
\end{array}\right.
$$

HSI and HSV have the same definition of hue, but the other components differ. The values for $\mathrm{s}$ and $\mathrm{v}$ of an HSV color are defined as follows:

$$
\begin{aligned}
& s=\left\{\begin{array}{cl}
0, & \text { if } \max =0 \\
\frac{\max -\min }{\max }=1-\frac{\min }{\max }, & \text { otherwise }
\end{array}\right. \\
& v=\max
\end{aligned}
$$

The HSV color space [2] is a popular choice for manipulating color. The HSV color space is developed to provide an intuitive representation of color and to approximate the way in which humans perceive and manipulate color. RGB to HSV is a nonlinear, but reversible, transformation. The hue (H) represents the dominant spectral component-color in its pure form, as in green, red, or yellow.

\section{YCbCr Color Model}

$\mathrm{RGB}$ to $\mathrm{YCbCr}$ [2] conversion is the most commonly used color coordinate system for the compression of image and video signals. $\mathrm{Y}$ is the luminance component and $\mathrm{Cb}$ and $\mathrm{Cr}$ are the chrominance components. The primary red, green, blue inputs $(\mathrm{R}, \mathrm{G}$, and $\mathrm{B})$ can be converted as follows:

$$
\begin{aligned}
& Y=0.299 * R+0.587 * G+0.114^{*} B \\
& C b=-0.169 * R-0.331 * G+0.500 * B \\
& C r=0.500 * R-0.419 * G-0.081 * B
\end{aligned}
$$

\section{PROPOSED CBIR}

In this paper, we discuss the design and implementation concept of multi color spaces used in our CBIR system for picture libraries. An experimental system, the SUCCE-SeR (Simple and Useful Multi Color Concepts for Effective Search and Retrieval) system, has been developed to validate the methods.

\section{A. System Architecture}

The generalized architecture of the SUCCESeR retrieval system is presented in Fig. 2. Consider s number of color space with $\mathrm{p}$ number of color planes in each color space. In each color plane nxn square sub blocks are constructed. There are $b$ sub blocks in each p plane. On every b sub blocks the selected energy compaction transform is applied.

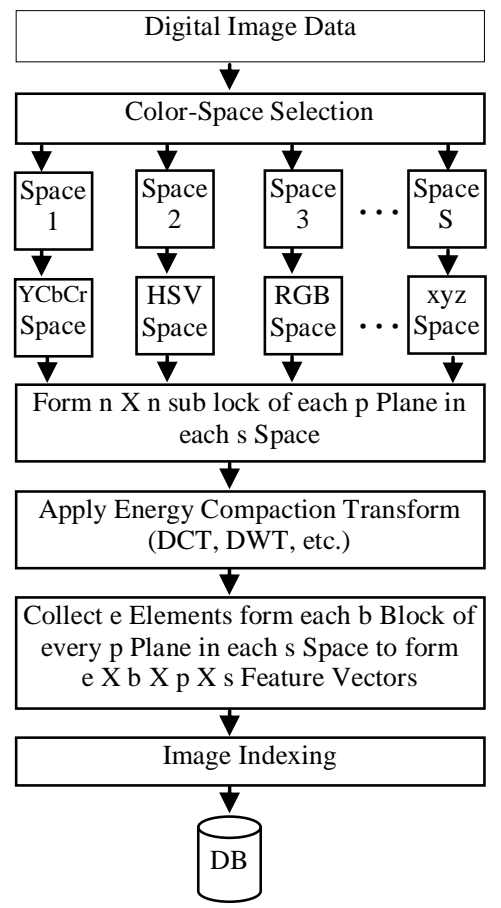

Figure 2. The architecture of feature indexing process

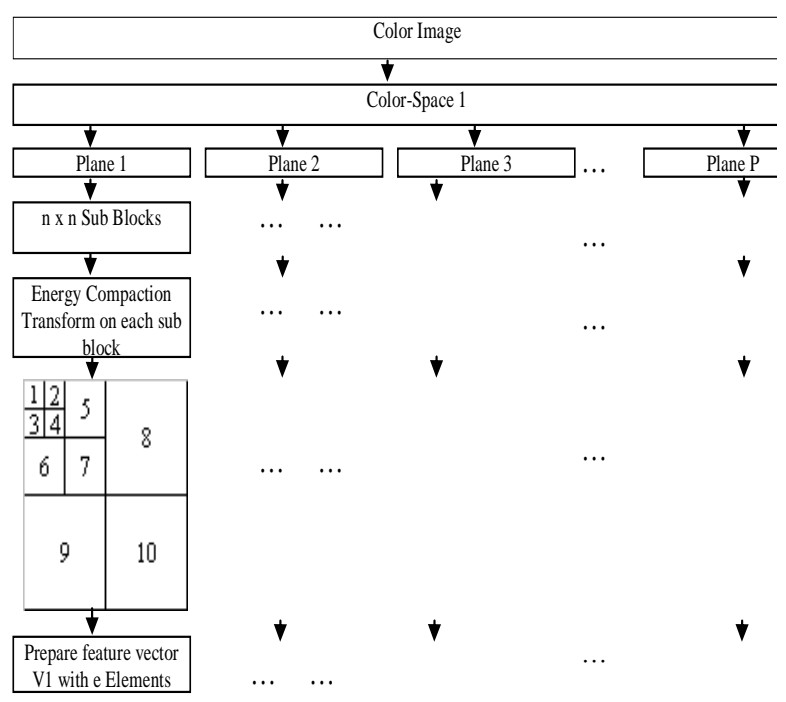

Figure 3. The plane-wise architecture of feature indexing process

The e number of elements in each $b$ sub block is considered to form feature vector. So, the feature vector can be form by selecting e elements from each $b$ sub blocks of each $p$ plane in every s space. The different set of e elements is formed to test the retrieval results. The interface program helps to select different elements for each color space to check the retrieval results. For different color space the procedure remains the same. The procedure is presented in Fig. 3.

For a given color space, the feature vector V1 can be formed by in manner given in Fig. 3. 


\section{B. Feature Extraction and Indexing}

To be precise the different color models used for extracting features in SUCCESeR system are RGB, HSV and $\mathrm{YCbCr}$. As we know there are three color planes in each color space. Energy in image is due to color component. The energy of the image is extracted using discrete cosine transform (DCT). Discrete cosine transform is applied on each $8 \times 8 \mathrm{sub}$ blocks of each color plane in each color space of every image in the database. The maximum energy of the image is concentrated in DC component of DCT and it reduces as we move down diagonally in each sub block.

\begin{tabular}{|c|c|c|c|}
\hline 1 & 2 & \multirow{2}{*}{5} & \multirow{3}{*}{8} \\
\hline 3 & 4 & & \\
\hline \multicolumn{2}{|c|}{6} & 7 & \\
\hline \multicolumn{3}{|c|}{9} & 10 \\
\hline
\end{tabular}

Figure 4. The 10 elements in of a $8 \times 8$ DCT coefficient

The 10 compiled elements shown in Fig. 4 from each $8 \times 8$ DCT coefficients for each color plane are used in the process of forming feature vectors. Two feature vectors are used to for image indexing. The first feature vector is formed by selecting only one element i.e. first element in each color plane; the second feature vector is formed by selecting first four elements of each color plane, and so on. This is shown in Fig. 5. To understand better the above concept is put in another form which is given in Fig. 6.

\begin{tabular}{|c|c|c|c|c|c|c|}
\hline \multirow{3}{*}{$\begin{array}{l}\text { Vector } \\
\mathrm{V}_{111}\end{array}$} & \multirow{3}{*}{\begin{tabular}{l}
\multicolumn{1}{c}{ Description of Vectors } \\
Vector elements include one \\
coefficient form every $8 \times 8$ sub- \\
block in each color space
\end{tabular}} & \multicolumn{5}{|c|}{ Color Space } \\
\hline & & RGB & \multicolumn{2}{|c|}{ HSV } & \multicolumn{2}{|c|}{$\mathrm{YCbCr}$} \\
\hline & & 1 & 1 & & 1 & \\
\hline \multirow{2}{*}{$\mathrm{V}_{112}$} & \multirow{2}{*}{$\begin{array}{l}\text { Vector elements include one } \\
\text { coefficient in RGB and } \mathrm{HSV} \text {, and } \\
\text { two coefficient in } \mathrm{YCbCr} \text { form } \\
\text { every } 8 \times 8 \text { sub-block }\end{array}$} & 1 & 1 & & 1 & 2 \\
\hline & & & & & & \\
\hline \multirow{2}{*}{$\mathrm{V}_{121}$} & \multirow{2}{*}{$\begin{array}{l}\text { Vector elements include one } \\
\text { coefficient in RGB and YCbCr, } \\
\text { and two coefficient in HSV form } \\
\text { every } 8 \times 8 \text { sub-block }\end{array}$} & 1 & 1 & 2 & 1 & \\
\hline & & & & & & \\
\hline \multirow{2}{*}{$\mathrm{V}_{122}$} & \multirow{2}{*}{$\begin{array}{l}\text { Vector elements include one } \\
\text { coefficient in RGB, and two } \\
\text { coefficient in } \mathrm{HSV} \text { and } \mathrm{YCbCr} \\
\text { form every } 8 \times 8 \text { sub-block }\end{array}$} & \multirow[t]{2}{*}{1} & 1 & 2 & 1 & 2 \\
\hline & & & & & & \\
\hline
\end{tabular}

Figure 5. Description of elements in a vector

\begin{tabular}{|c|c|c|c|c|c|c|c|c|c|c|}
\hline & \multicolumn{9}{|c|}{$\begin{array}{c}\text { Number of Elements Used in each Feature } \\
\text { Vector Formation (Out of 10 DCT } \\
\begin{array}{c}\text { Feature } \\
\text { Vector }\end{array}\end{array}$} & \multicolumn{3}{|c|}{$\begin{array}{c}\text { Number } \\
\text { of } \\
\text { of } \\
\text { Elements } \\
\text { in Each }\end{array}$} \\
\cline { 2 - 9 } & $\mathrm{r}$ & $\mathrm{g}$ & $\mathrm{b}$ & $\mathrm{h}$ & $\mathrm{s}$ & $\mathrm{v}$ & $\mathrm{y}$ & $\mathrm{cb}$ & $\mathrm{cr}$ & $\begin{array}{c}\text { Feature } \\
\text { Vector }\end{array}$ \\
\hline $\begin{array}{c}\text { Vector } \\
\mathrm{V}_{111}\end{array}$ & 1 & 1 & 1 & 1 & 1 & 1 & 1 & 1 & 1 & 9 \\
\hline $\begin{array}{c}\text { Vector } \\
\mathrm{V}_{112}\end{array}$ & 1 & 1 & 2 & 1 & 1 & 2 & 1 & 1 & 2 & 12 \\
\hline $\begin{array}{c}\text { Vector } \\
\mathrm{V}_{121}\end{array}$ & 1 & 2 & 1 & 1 & 2 & 1 & 1 & 2 & 1 & 12 \\
\hline $\begin{array}{c}\text { Vector } \\
\mathrm{V}_{122}\end{array}$ & 1 & 2 & 2 & 1 & 2 & 2 & 1 & 2 & 2 & 15 \\
\hline
\end{tabular}

Figure 6. The elements used in vector formation

\section{Equations}

The chi-square distance measure [17] between features of query object and database object is used in [18] to match and display images. It is given in (4).

$$
\text { dissimilarity }=\sum_{i=1}^{12}\left(\frac{F q(i)-F t(i)}{F q(i)+F t(i)}\right)^{2}
$$

The dissimilarities are sorted in descending order. Lower the dissimilarity closest the match. The closest matched images are our candidate images and they are displayed using proposed SUCCESeR System interface.

\section{RESUlt AND ANALYSIS}

In this paper, the implemented CBIR system using different color models can be deployed to retrieve images in picture libraries.

\section{A. Image Database}

The SUCCESeR system results were compared with the existing SIMPLIcity system results given in [7] which was already evaluated and compared with WBIIS [16] based on a same subset of the COREL database, formed by 10 image categories shown in Table I (a), each containing 100 pictures used in [7] and [16]. Within this database, it is known whether any two images are of the same category.

The SUCCESeR system was also evaluated based on a subset of the COREL database, formed by 10 image categories shown in Table I (b), each containing 100 pictures. In particular, a retrieved image is considered a match if and only if it is in the same category as the query. This assumption is reasonable since the 10 categories were chosen so that each depicts a distinct semantic topic. 
Table I. COREL Categories of Images Tested

(IJACSA) International Journal of Advanced Computer Science and Applications, Special Issue on Image Processing and Analysis

\begin{tabular}{|c|c|}
\hline ID & Category Name \\
\hline 1 & Africa people and villages \\
\hline 2 & Beach \\
\hline 3 & Landscape with buildings \\
\hline 4 & Buses \\
\hline 5 & Dinosaurs \\
\hline 6 & Elephants \\
\hline 7 & Flowers \\
\hline 8 & Horses \\
\hline 9 & Mountains and glaciers \\
\hline 10 & Food \\
\hline
\end{tabular}

(a)

\begin{tabular}{|c|c|}
\hline ID & Category Name \\
\hline 1 & Butterfly \\
\hline 2 & Beach \\
\hline 3 & Landscape with buildings \\
\hline 4 & Buses \\
\hline 5 & Dinosaurs \\
\hline 6 & Elephants \\
\hline 7 & Flowers \\
\hline 8 & Horses \\
\hline 9 & Mountains and glaciers \\
\hline 10 & Food \\
\hline
\end{tabular}

(b)

\section{B. Evaluation of the System}

To provide numerical results, we tested 30 sample images chosen randomly from ten categories, each containing three of the images. A retrieved image is considered a match if it belongs to the same category of the query image. The categories of images tested are listed in Table I. Most categories simply include images containing the specified objects. The precision is calculated using (5).

$$
\text { Precision }=\frac{\text { Number of relevant documents retrieved }}{\text { Total number of documents retrieved }}
$$

\section{Performance of SUCCESeR versus Simplicity}

For each of the ten image categories shown in Table I (a), the average precision performance comparison of our SUCCESeR system (using feature vector V111) with existing SIMPLIcity [7] system based on the three sample images are plotted in Fig. 7.

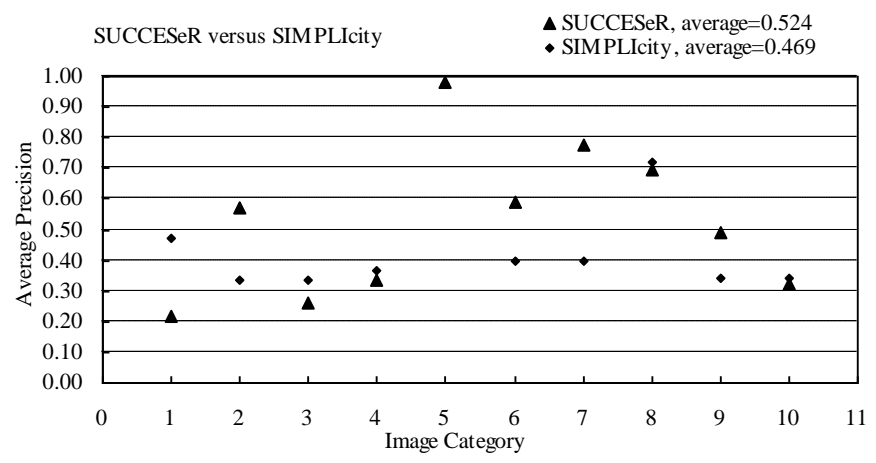

Figure 7. The average precision using vector $\mathrm{V}_{111}$

For each of the ten image categories shown in Table I (a), the average precision comparison based on the three sample images using feature vectors V112 and V122 are also plotted in Fig. 8 and Fig. 9 respectively.

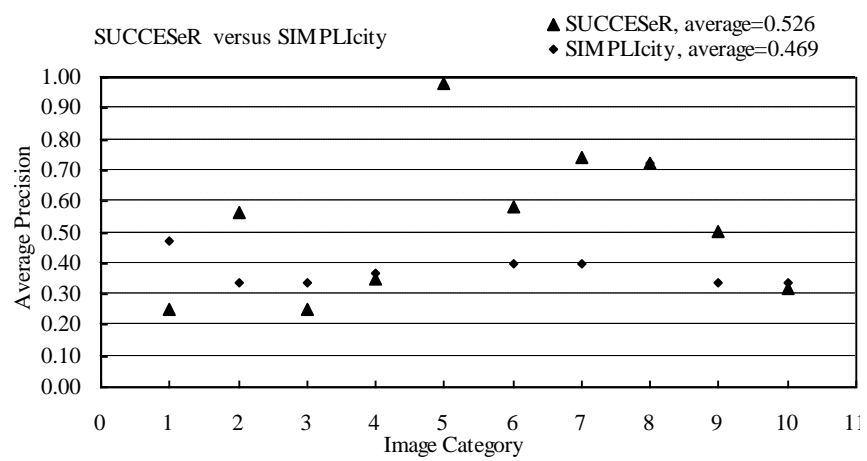

Figure 9. The average precision using vector $\mathrm{V}_{122}$

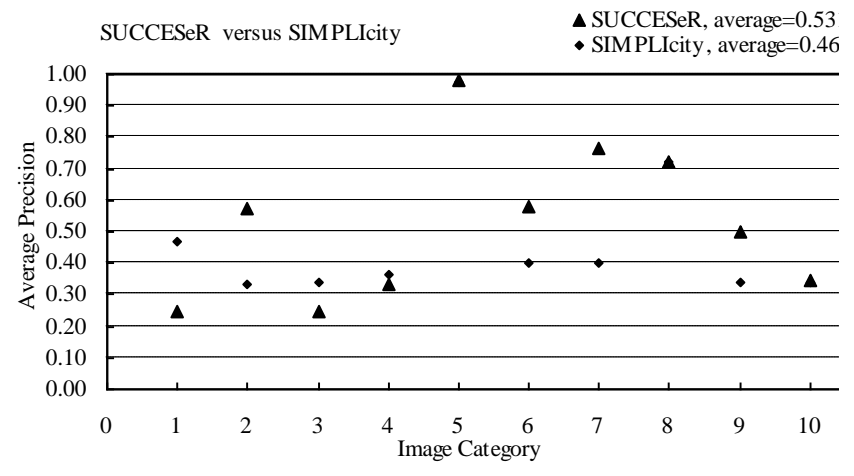

Figure 8. The average precision using vector $\mathrm{V}_{112}$

D. Performance of SUCCESeR

The retrieval results with different set of image categories in a given database are also comparable with existing

SIMPLIcity [7] and WBIIS [16] systems.

Table II. COREL Categories of Images Tested

\begin{tabular}{|c|c|c|c|}
\hline \multirow{2}{*}{$\begin{array}{c}\text { Class } \\
\text { ID }\end{array}$} & \multicolumn{3}{|c|}{ Average Precision Using } \\
\cline { 2 - 4 } & $\begin{array}{c}\text { Feature Vector } \\
\mathrm{V}_{111}\end{array}$ & $\begin{array}{c}\text { Feature Vector } \\
\mathrm{V}_{112}\end{array}$ & $\begin{array}{c}\text { Feature Vector } \\
\mathrm{V}_{122}\end{array}$ \\
\hline 1 & 0.59 & 0.59 & 0.25 \\
\hline 2 & 0.53 & 0.55 & 0.56 \\
\hline 3 & 0.23 & 0.21 & 0.25 \\
\hline 4 & 0.32 & 0.31 & 0.35 \\
\hline 5 & 0.98 & 0.98 & 0.98 \\
\hline 6 & 0.49 & 0.48 & 0.58 \\
\hline 7 & 0.60 & 0.59 & 0.74 \\
\hline 8 & 0.65 & 0.63 & 0.72 \\
\hline 9 & 0.46 & 0.49 & 0.50 \\
\hline 10 & 0.29 & 0.27 & 0.32 \\
\hline Average & 0.514 & 0.510 & 0.526 \\
\hline
\end{tabular}

Beside the performance comparison of our SUCCESeR system (using feature vectors V111, V112 and V122) for each of the ten image categories shown in Table I (a), the average precision based on the three sample images using SUCCESeR feature vectors V111, V112, and V122 for each of the ten 
image categories shown in Table I (b) were also measured and it is given in Table II.

The performance in terms of the average precision using graph is also shown in Fig. 10, Fig 11 and Fig. 12 for each of the ten image categories shown in Table I (b) based on the three sample images using SUCCESeR feature vectors V111, V112, and V122.

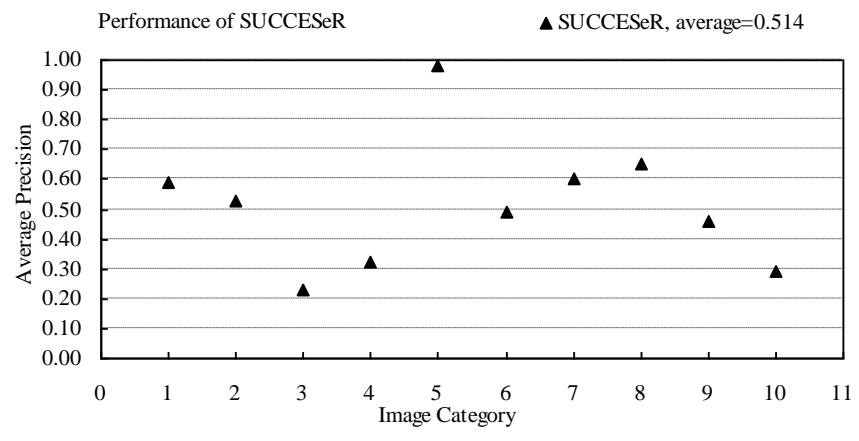

Figure 10. The average precision using vector $\mathrm{V}_{111}$

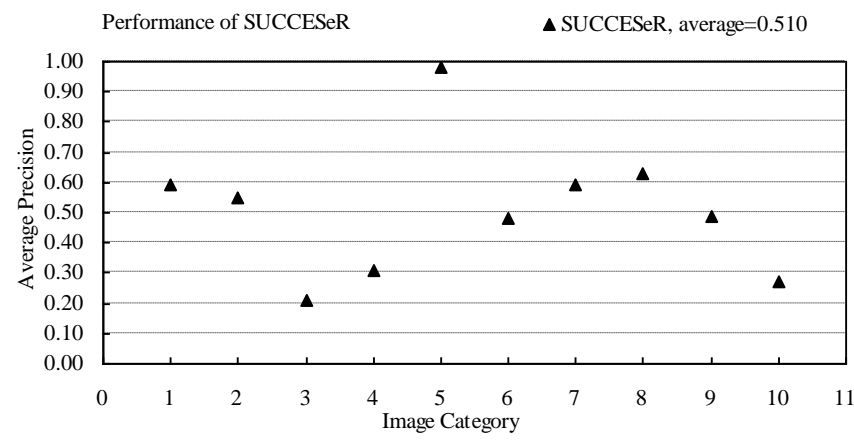

Figure 11. The average precision using vector $\mathrm{V}_{121}$

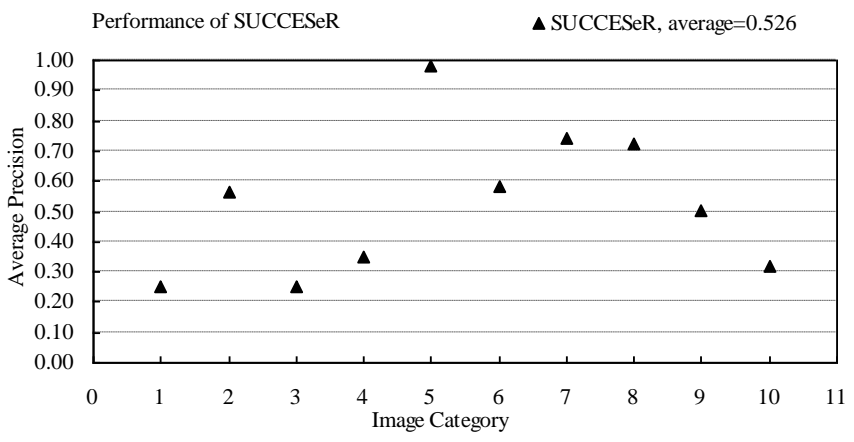

Figure 12. The average precision using vector $\mathrm{V}_{122}$

\section{E. Performance of SUCCESeR in terms of Speed}

The algorithm has been implemented on a Pentium IV $2.4 \mathrm{GHz}$ PC using the Win-dows XP operating system. To compute the feature vectors for the 1000 color images of size $384 \times 256$ resized to $80 \times 80$ to increase speed in our generalpurpose image database requires approximately 294.598 seconds for indexing images. The indexing using feature vector V111 takes less time as compared to feature vector V122.
The matching speed is very fast. When the query image is given form the database, it takes about 2.5:2.9 seconds of CPU time on average to extract and display all the 20 images in the indexed 1000-image database using the similarity measure given in (5).

\section{F. Retrieval Results}

The query image is the image at the upper-left corner. The numbers below the images are the ID numbers of the images in the database. The other numbers next to image ID are the values of the similarity measure given in (5) between the query image and the matched image.

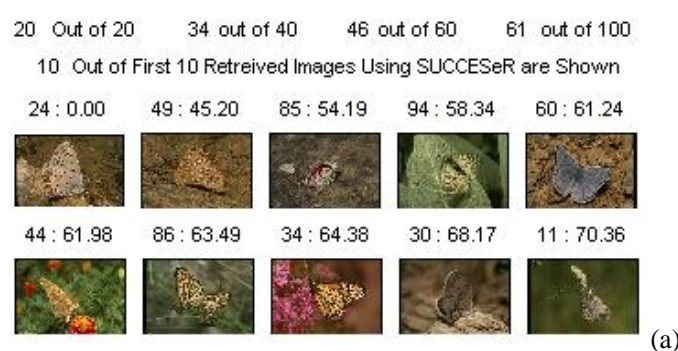

(a)
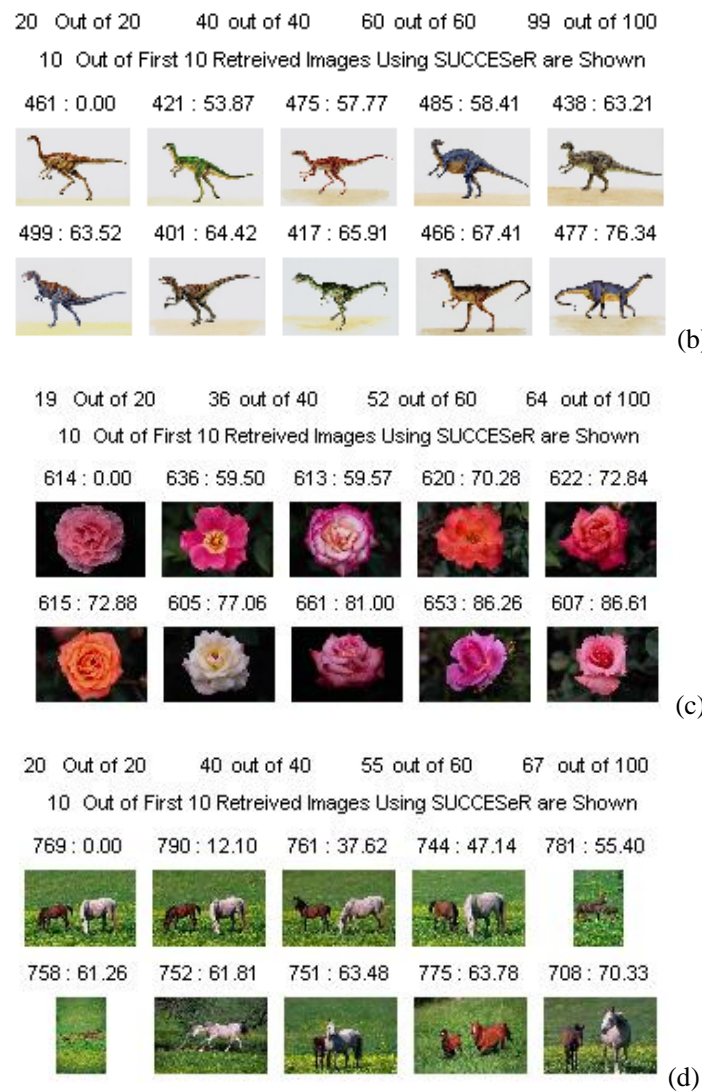

Figure 13. Retrieval Results using feature vector $\mathrm{V}_{111}$ (a) Butterfly (b) Dinosaurs (c) Flowers and (d) Horses.

The precision within the first 20 retrieved images are calculated. However the precision within the first 40, 60 and 100 retrieved images are also displayed.

There is no big difference between the retrieval results using the feature vector V111 and feature vector V122. The results using feature vector V111 using our SUCCESeR 
program interfaces are shown in Fig. 13. The results using feature vector V122 using our SUCCESeR program interfaces are shown in Fig. 14.
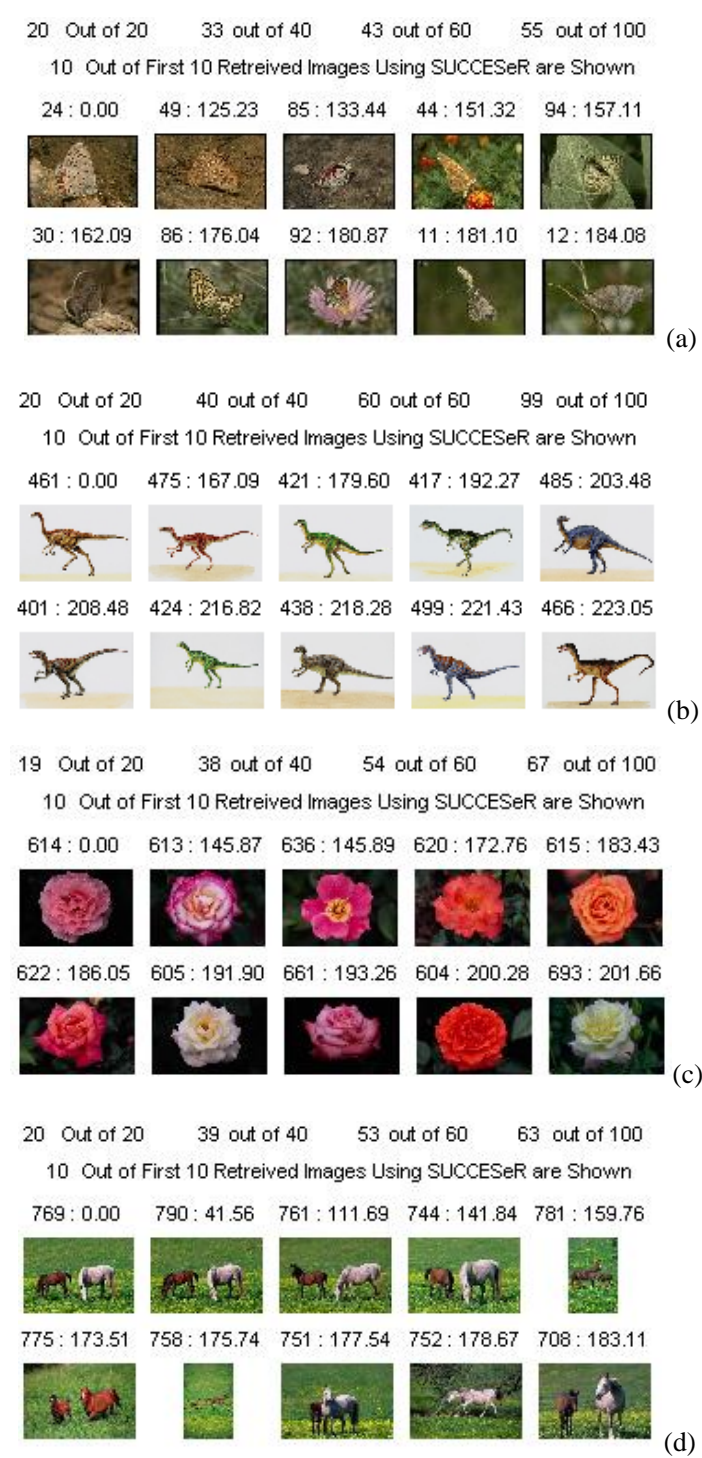

Figure 14. Retrieval Results using feature vector $\mathrm{V}_{122}$ (a) Butterfly (b) Dinosaurs (c) Flowers and (d) Horses.

\section{SUMMARY AND CONCLUSION}

The SUCCESeR system gives average precision 1 within first 10 retrieved images with butterfly, beach, dinosaurs, flowers, and food image categories. The average precision within first 20 retrieved images with butterfly, beach, dinosaurs, flowers, and food image categories also tends to 1. The indexing using feature vector V111 takes less time as compared to feature vector V122.

\section{FUTURE SCOPE}

One possible solution to improve the results could be of considering the combination of other color models. Again this may take more time for indexing images. Also the results can be improved by including more elements in a new feature vectors than the elements in feature vector V122. Again this may take more time for indexing images and retrieval. Here, the elements in feature vector V111 and feature vector V122 are 9 and 15 respectively.

\section{ACKNOWLEDGMENT}

We propose our sincere thanks to one and all those who have helped and encouraged us to think in a possible direction to work and devise a useful method that helps our society to move one step ahead.

\section{REFERENCES}

[1] Swain, M. J. \& Ballard, D. H. (1991) Color Indexing, International Journal of Computer Vision, 7, 1, 11-32.

[2] B. S. Manjunath, Jens-Rainer Ohm, Vinod V. Vasudevan, and AkioYamada, "Color and Texture Descriptors". In: IEEE Transactions on Circuits and Systems for Video Technology, Vol. 11, No. 6, June 2001, pp. 70-715.

[3] Remco C. Veltcamp, Mirela Tanse, "Content Based Image Retrieval Systems". A Survey, Technical Report UU-CS-2000-34, October 2000, pp. 1-62.

[4] Yining Deng, B. S. Manjunath, Charles Kenney, Michael S. Moore, Student Member, Hyundoo Shin, "An Efficient Color Representation for Image Retrieval" IEEE Transactions on Image Processing, 2001.

[5] J. R. Smith and S.-F. Chang. "Tools and techniques for color image retrieval", In Symposium on Electronic Imaging: Science and Technology - Storage \& Retrieval for Image and Video Databases IV, volume 2670, San Jose, CA, February 1996. IS\&T/SPIE.

[6] James Hafner, Harpreet S.Sawhney, Will Equits, Myron Flickner and Wayne Niblack, "Efficient Color Histogram Indexing for Quadratic Form Distance Functions", IEEE Trans. on Pattern Analysis and Machine Intelligence, Vol. 17, No. 7, July 1995.

[7] James Z. Wang, Jia Li, Gio Wiederhold, "SIMPLIcity: Semanticssensitive Integrated Matching for Picture LIbraries," IEEE Trans. on Pattern Analysis and Machine Intelligence, vol 23, no.9, pp. 947-963, 2001.

[8] S. Nandagopalan, Dr. B. S. Adiga, and N. Deepak, "A Universal Model for Content-Based Image Retrieval," PWASET Vol. 36, December 2008, ISSN 2070-3740.

[9] P. S. Hiremath, Jagadeesh Pujari, "Content Based Image Retrieval using Color, Texture and Shape features," 15th International Conference on Advanced Computing and Communications, IEEE Computer Society 2007, pp. 780-784.

[10] Se-Hwan Kim, Woontack Woo and Yo-Sung Ho, "Image Retreival Using Multi-Scale Color Clustering," IEEE, 2001, pp. 666-669.

[11] J. Geusebroek, D. Koelma, A.W.M. Smeulders and T. Gevers, "Image Retrieval and Segmentation based on Color Invariants," IEEE, pp. 1-2, 2000.

[12] H. Nezamabadi-pour and S. Saryazdi, "Object-Based Image Indexing and Retrieval in DCT Domain using Clustering Techniques," PWASET Vol. 3, January 2005, ISSN 1307-6884, pp.98-101

[13] C.W.Ngo, T.C.Pong and R.T.Chin, "Exploiting image indexing techniques in DCT domain", pattern Recognition, 2001, vol. 34, pp. 1841-1851.

[14] G.Feng and J.Jiang, "JPEG compressed image retrieval via statistical features", Pattern Recognition, 2003, vol. 36, pp. 977-985.

[15] A.Vailaya, A.K.Jain and H.J.Zhang, "On image classification: city vs.landscape”, Pattern Recognition, 1998, vol. 31, pp. 1921-1935.

[16] J. Z. Wang, G. Wiederhold, O. Firschein, and X.W. Sha, "Content-Based Image Indexing and Searching Using Daubechies' Wavelets," Int'l J. Digital Libraries, vol. 1, no. 4, pp. 311-328, 1998.

[17] Text of ISO/IEC 15 938-3 Multimedia Content Description InterfacePart 3: Visual. Final Committee Draft, ISO/IEC/JTC1/SC29/WG11, Doc. N4062, Mar. 2001.

[18] Roger Weber and Michael Mlivoncic, "Efficient Region-Based Image Retrieval", ACM CIKM '03 November 3-8, 2003, USA.

[19] M. Flickner, H. Sawhney, W. Niblack, J. Ashley, Q. Huang, B. Dom et al. "Query by Image and Video Content: The QBIC System," IEEE Computer, vol. 28, no. 9, 1995. 
[20] J.R. Smith and C.S. Li, "Image Classification and Querying Using Composite Region Templates," Int'l J. Computer Vision and Image Understanding, vol. 75, nos. 1-2, pp. 165-174, 1999.

[21] A. Gupta and R. Jain, "Visual Information Retrieval," Comm. ACM, vol. 40, no. 5, pp. 70-79, May 1997.

[22] S. Mukherjea, K. Hirata, and Y. Hara, "AMORE: A World Wide Web Image Retrieval Wngine," Proc. World Wide Web, vol. 2, no. 3, pp. 115-132, 1999.

[23] S. Stevens, M. Christel, and H. Wactlar, "Informedia: Improving Access to Digital Video," Interactions, vol. 1, no. 4, pp. 67-71, 1994.

[24] A. Pentland, R.W. Picard, and S. Sclaroff, "Photobook: Tools for Content-Based Manipulation of Image Databases," Proc. SPIE, vol. 2185, pp. 34-47, Feb. 1994.

[25] R.W. Picard and T. Kabir, "Finding Similar Patterns in Large Image Databases, ${ }^{\circ}$ Proc. IEEE Int'l Conf. Acoustics, Speech, and Signal Processing, vol. 5, pp. 161-164, 1993.

[26] T.P. Minka and R.W. Picard," Interactive Learning Using a Society of Models, ${ }^{\circ}$ Pattern Recognition, vol. 30, no. 3, p. 565, 1997.

[27] J.Z. Wang, J. Li, R.M. Gray, and G. Wiederhold, "Unsupervised Multiresolution Segmentation for Images with Low Depth of Field," IEEE Trans. Pattern Analysis and Machine Intelligence, vol. 23, no. 1, pp. 85-91, Jan. 2001.

[28] S. Stevens, M. Christel, and H. Wactlar, "Informedia: Improving Access to Digital Video," Interactions, vol. 1, no. 4, pp. 67-71, 1994.

[29] W.Y. Ma and B. Manjunath, "NaTra: A Toolbox for Navigating Large Image Databases," Proc. IEEE Int'l Conf. Image Processing, pp. 568571, 1997.

[30] R. Jain, S.N.J. Murthy, P.L.-J. Chen, and S. Chatterjee, "Similarity Measures for Image Databases," Proc. SPIE, vol. 2420, pp. 58-65, Feb. 1995.

[31] E.G.M. Petrakis and A. Faloutsos, "Similarity Searching in Medical Image Databases," IEEE Trans. Knowledge and Data Eng., vol. 9, no. 3, pp. 435-447, May/June 1997.

[32] Y. Rubner, L.J. Guibas, and C. Tomasi, "The Earth Mover's Distance, Multi-Dimensional Scaling, and Color-Based Image Retrieval," Proc. DARPA Image Understanding Workshop, pp. 661- 668, May 1997.
[33] B. M. Mehtre, M. S. Kankanhalli, A. D. Narasimhalu and G. C. Man, "Color matching for image retrieval," Pattern Recognition Letters, Vol. 16, pp. 325-331, March 1995.

\section{AUTHORS PROFILE}

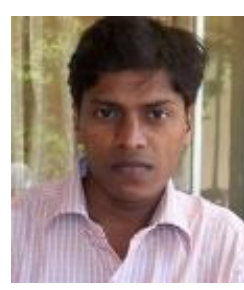

Satishkumar L. Varma received B.Tech. and M.Tech. degrees from Dr. Babasaheb Ambedker Technological University, Lonere, Maharashtra, India in June 2000 and January 2004 respectively. He is pursuing Ph.D. degree from the SRTM University, Nanded, India. He has also worked with DBIT as an Assistant Professor and Head in the department of Information Technology. Currently he is Assistance Professor in the department of Computer Engineering in Pillai's Institute of Information Technology, New Mumbai, India. He has published one Journal paper and more than 12 papers in referred National as well as International Conferences including IEEE, Springer and IET. His research interest includes Multimedia Systems, Digital Image and Video Processing and Computer Vision.

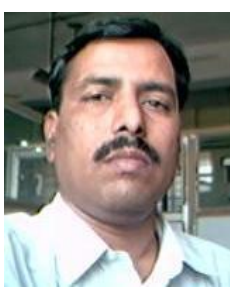

Sanjay N. Talbar received his B.E and M.E degrees from SGGS Institute of Technology, Nanded, India in 1985 and 1990 respectively. He obtained his $\mathrm{PhD}$ from SRTM University, Nanded, India in 2000. He received the "Young Scientist Award" by URSI, Italy in 2003. He had Collaborative research programme at Cardiff University Wales, UK. Presently he is working as Professor and Head, Department of Electronics \& Telecommunication Engg., SGGS Institute of Engineering \& Technology Nanded, India.

He has published 12 journal papers and more than 65 papers in referred National as well as International Conferences. His research interests includes Image processing, Multimedia Computing and Embedded System Design. He is a member of many prestigious committees in academic field of India. 